\title{
Fluctuation Theorem for a Small Engine and Magnetization Switching by Spin Torque
}

\author{
Yasuhiro Utsumi \\ Department of Physics Engineering, Faculty of Engineering, Mie University, Tsu, Mie, 514-8507, Japan \\ Tomohiro Taniguchi \\ National Institute of Advanced Industrial Science and Technology (AIST), \\ Spintronics Research Center, 1-1-1 Umezono, Tsukuba 305-8568, Japan
}

(Dated: June 25, 2021)

\begin{abstract}
We consider a reversal of the magnetic moment of a nano-magnet by the fluctuating spin-torque induced by a non-equilibrium current of electron spins. This is an example of the problem of the escape of a particle from a metastable state subjected to a fluctuating non-conservative force. The spin-torque is the non-conservative force and its fluctuations are beyond the description of the fluctuation-dissipation theorem. We estimate the joint probability distribution of work done by the spin torque and the Joule heat generated by the current, which satisfies the fluctuation theorem for a small engine. We predict a threshold voltage above which the spin-torque shot noise induces probabilistic switching events and below which such events are blocked. We adopt the theory of the full-counting statistics under the adiabatic pumping of spin angular momentum. This enables us to account for the backaction effect, which is crucial to maintain consistency with the fluctuation theorem.

PACS numbers: 05.40.-a, 72.70.+m, 75.60.Jk, 72.25.-b
\end{abstract}

The thermodynamics of small systems, the stochastic thermodynamics [1], is of growing importance in nanoscience. The key ingredient is the fluctuation theorem (FT) [1 3], which has been applied to the solid state physics recently and extends the fluctuation-dissipation theorem as well as the Onsager relations far from equilibrium (see e.g. Refs. 2-9). Recent studies suggest that the FT is also useful to analyze small engines [10 12]. In a small engine, during a short time step $\Delta t$ at finite temperature $T$, the input heat $q$ and the output work $w$ fluctuate and can take positive and negative values [Fig. [1(a)]. The FT ensures that the joint probability distribution satisfies

$$
P_{R, \Delta t}(-q,-w)=P_{\Delta t}(q, w) e^{-\beta(q+w)}, \beta=\left(k_{\mathrm{B}} T\right)^{-1},
$$

where the subscript $R$ indicates that the external driving is reversed. From Jensen's inequality, this equation reproduces the Carnot theorem, $\langle w\rangle /\langle q\rangle \leq 1$. The FT (1) is applicable even when a cycle is not defined. The work can be attributed to a non-conservative force originating from a heat flow between two baths [Fig. 1 (a)]. Let us couple the small engine to a small system. The energy variation of the small system is equal to the fluctuating work:

$$
\Delta E=w .
$$

We expect that Eqs. (1) and (2) are applicable to a wide spectrum of mesoscopic systems driven by nonconservative forces.

In the present paper, we apply this idea to the problem of the escape of a particle from a metastable state [13. subjected to a fluctuating non-conservative force. We consider the following specific setup: a nano-magnet connected to a left ferromagnetic lead (source) and a right normal metal lead (drain) [Fig. 1 (b)]. The magnetization vector of the bulk left ferromagnetic lead $\mathbf{M}_{L}$ is fixed. Let us assume that the magnetization of the nanomagnet $\mathbf{M}$ is anti-parallel to $\mathbf{M}_{L}$. By applying a sourcedrain bias voltage $V$, spin polarized electrons are injected from the ferromagnetic lead, which exert a torque on the nano-magnet [14]. When the magnetic moment $\mathbf{M} \mathcal{V}(\mathcal{V}$ is the volume of the nano-magnet) is small, above a critical voltage $V^{*}, \mathbf{M}$ is reversed and aligns parallel to $\mathbf{M}_{L}$. The spin-torque is generated by the non-equilibrium current and thus the non-conservative force. It performs the work $w$ on the small system (the nano-magnet) and is accompanied by the Joule heat $q$. Since the spin angular momentum exchanged between electrons and the nanomagnet is discretized by $\hbar$, the spin-torque fluctuates and even under the critical voltage $V^{*}$, it can switch the magnetic moment probabilistically. The exponent $\Delta$ of the switching probability

$$
P_{\tau} \sim e^{-\Delta},
$$

is well studied for equilibrium thermal fluctuations, which are Gaussian-distributed (see e.g. Refs. $15-18$ and references therein). However, this is not the case for the non-equilibrium fluctuations. In current experiments 19], an MgO-insulating tunnel barrier is sandwiched between the nano-magnet and the ferromagnetic lead, which generates a Poisson-distributed shotnoise out of equilibrium 20. Previous studies analyzing the non-equilibrium spin-torque shot noise [21 23] limited themselves to the Gaussian fluctuations. The nonGaussian fluctuations are beyond the description of the fluctuation-dissipation theorem and, to our knowledge, have not been reliably described.

In the present paper, we determine the distribution 
of non-Gaussian fluctuations by using the full-counting statistics [24] under the adiabatic pumping [25, 26], which gives the joint probability distribution consistent with the FT for a small engine (11). We evaluate the switching exponent $\Delta$ and predict another threshold voltage $V_{\text {th }}$ under which the probabilistic switching is completely blocked. This is a result of the backaction, i.e., the adiabatic pumping of the spin angular momentum [27], as a consequence of the FT.

Langevin equation in the energy coordinate - We take the $z$-axis parallel to the direction of the left magnetization, $\mathbf{e}_{z}=(0,0,1)=\mathbf{M}_{L} /\left|\mathbf{M}_{L}\right|$, which is fixed [Fig. [1 (b)]. We assume the uniaxial anisotropy of the nanomagnet in the $z$-direction. The anisotropic energy is,

$$
E=-\frac{M H_{K} \mathcal{V}\left(\mathbf{e}_{z} \cdot \mathbf{m}\right)^{2}}{2}=-\frac{M H_{K} \mathcal{V} \cos ^{2} \theta}{2},
$$

where $M=|\mathbf{M}|$ is the saturation magnetization and $\mathbf{m}=\mathbf{M} / M$. In the spherical coordinates, it is expressed as $\mathbf{m}=(\sin \theta \cos \phi, \sin \theta \sin \phi, \cos \theta)$. The anisotropic magnetic field is typically $H_{K}>0$, and thus the magnetic moment tends to align with $\mathbf{m}=\mathbf{e}_{z}$ or $\mathbf{m}=-\mathbf{e}_{z}$. These 2 states are separated by the energy barrier $M H_{K} \mathcal{V} / 2$. Because of this bistability, the setup is applicable to a memory device [19].

The dynamics of the nano-magnet is described by the stochastic Landau-Lifshitz-Gilbert equation,

$$
\dot{\mathbf{m}}=-\gamma \mathbf{m} \times\left(\mathbf{H}_{\mathrm{eff}}+\mathbf{h}\right)+\alpha \mathbf{m} \times \dot{\mathbf{m}}-\gamma \mathbf{I}_{S} /(M \mathcal{V}),
$$

where $\gamma=2 \mu_{\mathrm{B}} / \hbar$ is the gyromagnetic ratio and $\mu_{\mathrm{B}}$ is the Bohr magneton. The effective magnetic field is $\mathbf{H}_{\text {eff }}=$ $-\mathcal{V}^{-1} \partial E / \partial \mathbf{M}=H_{K} \cos \theta \mathbf{e}_{z}$, and $\mathbf{h}$ is its fluctuation induced by thermally excited magnons. It is a Gaussian white noise, i.e., $\left\langle h_{j}(t)\right\rangle=0(j=x, y, z)$, and the correlation is instantaneous and isotropic: $\left\langle h_{j}(t) h_{k}\left(t^{\prime}\right)\right\rangle=$ $2 \alpha k_{\mathrm{B}} T \delta_{j k} \delta\left(t-t^{\prime}\right) /(\gamma M \mathcal{V})$. The Gilbert damping constant $\alpha$ also appears in the second term of the lhs of Eq. (5), indicating the relaxation to $\mathbf{m}=\mathbf{e}_{z}$ or $\mathbf{m}=-\mathbf{e}_{z}$. The spin-torque $\mathbf{I}_{S}=\mathcal{I} \mathbf{m} \times\left(\mathbf{e}_{z} \times \mathbf{m}\right)$ aligns $\mathbf{M}$ parallel to $\mathbf{M}_{L}$ [14].

Since typically the damping and the spin torque are weak, the variation of the energy after a single precession is small [15, 17, 18]. Therefore, the magnetic moment precesses along the $z$ axis with the frequency $\dot{\phi}=\gamma H_{K} \cos \theta \equiv \Omega$ along a constant energy trajectory given by Eq. (4). In the following, we will concentrate on the negative branch, $\Omega=-\sqrt{-2 \gamma^{2} H_{K} E /(M \mathcal{V})}$, i.e., $-1 \leq m_{z} \leq 0$. It is convenient to consider the time derivative of the energy (4) averaged over a single precession: $\overline{\dot{E}(t)}=\Omega \int_{t}^{t+2 \pi / \Omega} d t^{\prime} \dot{E}\left(t^{\prime}\right) /(2 \pi)$. In the first order in $\alpha$ and $\mathcal{I}$, we obtain Eq. (2) for our system:

$$
\overline{\dot{E}}=\overline{\dot{\mathbf{M}} \cdot \partial E / \partial \mathbf{M}}=\overline{p_{S}}-\overline{p_{\alpha}},
$$

where $p_{\alpha}=\gamma M \mathcal{V}\left(\mathbf{m} \times \mathbf{H}_{\text {eff }}\right) \cdot\left(\alpha \mathbf{m} \times \mathbf{H}_{\text {eff }}+\mathbf{h}\right)$ is the sum of the power dissipated by the Gilbert damping
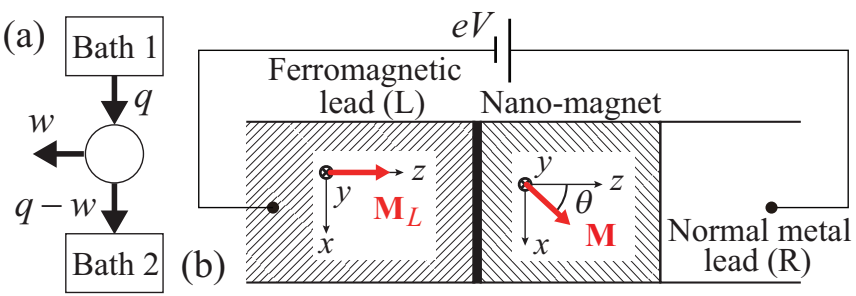

FIG. 1: (a) Schematic picture of a small engine. The input heat $q$ and the output work $w$ fluctuate. (b) A nano-magnet coupled to the left ferromagnetic lead and the right normal metal lead. The directions of magnetic moments of the ferromagnetic lead and the nano-magnet are $\mathbf{e}_{z}=(0,0,1)$ and $\mathbf{m}=(\sin \theta \cos \phi, \sin \theta \sin \phi, \cos \theta)$.

and that generated by the thermally fluctuating magnetic field. The average is $\left\langle\overline{p_{\alpha}}\right\rangle=G_{\alpha}(\hbar \Omega)^{2} \sin ^{2} \theta$, where $G_{\alpha}=\pi \alpha M \mathcal{V} /\left(h \mu_{\mathrm{B}}\right)$. The variance is proportional to the temperature times the average $\left\langle\delta \overline{p_{\alpha}(t)} \delta \overline{p_{\alpha}\left(t^{\prime}\right)}\right\rangle=$ $2 k_{\mathrm{B}} T\left\langle\overline{p_{\alpha}}\right\rangle \delta\left(t-t^{\prime}\right)\left(\delta \overline{p_{\alpha}} \equiv \overline{p_{\alpha}}-\left\langle\overline{p_{\alpha}}\right\rangle\right)$, which is a consequence of the fluctuation-dissipation theorem [28].

The power gain by the spin-torque is

$$
\overline{p_{S}}=2 \mu_{B} \overline{\mathbf{I}_{S} \cdot \mathbf{H}_{\text {eff }}} / \hbar=\Omega \overline{I_{S z}} .
$$

For the uniaxial anisotropy case, only the $z$ component of the spin torque is necessary $\overline{I_{S z}}=\mathcal{I} \sin ^{2} \theta$. Our main task is to determine the probability distribution of the fluctuating $\overline{I_{S z}}$ to be consistent with the FT for a small engine (1).

Fluctuation theorem for non-conservative force - During a time interval $\Delta t$, which is short but sufficiently longer than the period of the precession $2 \pi / \Omega, n$ electrons are transmitted through the nano-magnet from left to right leads and the $s$ electron spins flip from $\uparrow$ to $\downarrow$. They are given by $n=\int_{t}^{t+\Delta t} d t^{\prime} \overline{I\left(t^{\prime}\right)} / e$, where $I$ is the charge current, and $s=\int_{t}^{t+\Delta t} d t^{\prime} \overline{I_{S z}\left(t^{\prime}\right)} / \hbar$. When the energy change is slow enough, we can calculate the joint probability distribution $P_{\Delta t}(n, s)$ using the full-counting statistics under the adiabatic pumping with the pumping frequency $\Omega[25,26]$. The scaled cumulant generating function (SCGF) $\mathcal{F}_{G}$ is introduced as

$$
\sum_{n, s} P_{\Delta t}(n, s ; \Omega) e^{i \lambda n+i \chi s} \approx e^{\Delta t \mathcal{F}_{G}(\lambda, \chi ; \Omega)},
$$

where $\lambda$ and $\chi$ are counting fields for the numbers of transmitted electrons and flipped spins. Electrons in the left ferromagnetic lead and those in the right metal lead obey the Fermi distribution: $f_{r}(E)=1 /\left[e^{\beta\left(E-\mu_{r}\right)}+1\right]$ $(r=L, R)$. In equilibrium, the chemical potentials are at the Fermi level $\mu_{L}=\mu_{R}=E_{\mathrm{F}}$. The source drain bias voltage $V$ shifts the chemical potential of the left lead as $\mu_{L}=E_{\mathrm{F}}+e V$.

For now, to keep the discussion simple and specific, we keep the general form of the SCGF under the adia- 
batic pumping later, Eq. (20), and assume that the nanomagnet is ferromagnetic-insulating, although the current experiments use an insulator/metallic ferromagnet nanostructure [19]. The SCGF acquires the bi-directional Poisson form [29]:

$$
\begin{aligned}
\mathcal{F}_{G}(\lambda, \chi ; \Omega) & =\sum_{\nu, \nu^{\prime}= \pm} \Gamma_{\nu \nu^{\prime}}(\Omega)\left(e^{i \nu \lambda+i \nu^{\prime} \chi}-1\right) \\
& +\sum_{ \pm} \Gamma_{ \pm}\left(e^{ \pm i \lambda}-1\right) .
\end{aligned}
$$

The first line corresponds to the spin-flip tunneling process. The tunneling rate is

$$
\Gamma_{\nu \nu^{\prime}}(\Omega)=\sin ^{2} \theta G_{\nu \nu^{\prime}} \frac{\nu e V-\nu^{\prime} \hbar \Omega}{1-e^{-\beta\left(\nu e V-\nu^{\prime} \hbar \Omega\right)}},
$$

where $G_{++}=G_{--}=G_{+}$and $G_{+-}=G_{-+}=G_{-}$are spin-flip tunnel conductances. Their dimension is $h^{-1}$ and $G_{+/-}$connects $L \uparrow / L \downarrow$ and $R \downarrow / R \uparrow$ states. The second line of Eq. (9) corresponds to the spin-preserving tunneling process.

$$
\begin{aligned}
\Gamma_{\nu}= & {\left[G_{\mathrm{P}} \cos ^{2}(\theta / 2)+G_{\mathrm{AP}} \sin ^{2}(\theta / 2)\right.} \\
& \left.-\sin ^{2} \theta\left(G_{+}+G_{-}\right)\right](\nu e V) /\left(1-e^{-\nu \beta e V}\right) .
\end{aligned}
$$

Similar to the free energy [30], from the derivative of the SCGF, we can calculate the charge/spin current. For example, we obtain the spin-valve expression [31],

$$
\frac{\langle\bar{I}\rangle}{e}=\left.\frac{\partial \mathcal{F}_{G}(\lambda, 0 ; 0)}{\partial(i \lambda)}\right|_{\lambda=0}=\left(G_{\mathrm{P}} \cos ^{2} \frac{\theta}{2}+G_{\mathrm{AP}} \sin ^{2} \frac{\theta}{2}\right) e V,
$$

where $G_{\mathrm{P}}$ and $G_{\mathrm{AP}}$ are conductances in parallel and antiparallel alignments.

The SCGF is symmetric under the time reversal in the backward driving $\Omega \rightarrow-\Omega$. It leads the spintronic FT [8, 9]:

$$
\mathcal{F}_{G}(\lambda, \chi ; \Omega)=\mathcal{F}_{G, R}(-\lambda+i \beta e V, \chi+i \beta \hbar \Omega ;-\Omega),
$$

where the subscript $R$ means that the magnetizations are also reversed, $\mathbf{M} \rightarrow-\mathbf{M}$ and $\mathbf{M}_{L} \rightarrow-\mathbf{M}_{L}$ (which results in $G_{+} \leftrightarrow G_{-}$). After the inverse Fourier transform and identifying the work as $w=s \hbar \Omega$ [see Eq. (7)] and the Joule heat as $q=n e V$, we obtain the FT for a small engine (11). Our SCGF (9) together with the Langevin equation in the energy coordinate (6) enables us to calculate the switching exponent consistent with the FT.

Magnetization switching - The average value of the power (7) is given by

$$
\left\langle\overline{p_{S}}(\Omega)\right\rangle=\left.\hbar \Omega \frac{\partial \mathcal{F}_{G}(0, \chi ; \Omega)}{\partial(i \chi)}\right|_{\chi=0}=\Omega I_{S z}^{\Omega=0}-p_{\text {pump }} .
$$

The first term is the power gain by the spin torque: $I_{S z}^{\Omega=0}=\hbar \sin ^{2} \theta\left(G_{+}-G_{-}\right) e V$. The second term is the power dissipation by the adiabatic pumping of spin angular momentum [27]: $p_{\text {pump }}=\sin ^{2} \theta(\hbar \Omega)^{2}\left(G_{+}+G_{-}\right)$, which accounts for the backaction effect. We assume that initially the magnetizations are in antiparallel alignment, $m_{z}=\cos \theta=-1$. Then for $G_{+}<G_{-}$, which means that the spin-flip process $L \downarrow \rightarrow R \uparrow$ is the majority process, at positive $\mathrm{eV}$, there exists a frequency $\Omega^{*}$ at which the power gain and the power dissipation balance: $\left\langle\overline{p_{S}}\left(\Omega^{*}\right)\right\rangle=\left\langle\overline{p_{\alpha}}\left(\Omega^{*}\right)\right\rangle$. The condition leads, $\hbar \Omega^{*}=\left(G_{+}-G_{-}\right) \mathrm{eV} /\left(G_{+}+G_{-}+G_{\alpha}\right)$. When the magnitude of the precession frequency at $m_{z}=-1,-\Omega=\gamma H_{K}$ becomes smaller than $-\Omega^{*}, m_{z}$ starts to increase to $m_{z}=0$ and eventually reaches $m_{z}=1$. The critical voltage $e V^{*}$ above which the magnetization is reversed even in the absence of thermal fluctuations and spin-torque shot noise is

$$
\frac{e V^{*}}{2 \mu_{\mathrm{B}} H_{K}}=\frac{G_{+}+G_{+}+G_{\alpha}}{G_{-}-G_{+}} .
$$

Since the spin-torque shot noise is intrinsic and remains even at zero temperature, the nano-magnet switches probabilistically under $e V^{*}$. A convenient way to calculate such switching probability is the path-integral approach of the Langevin equation (6) [32]. The switching probability $P_{\tau}$ is the conditional probability to find $m_{z}=-1\left(E=-M H_{K} \mathcal{V} / 2\right)$ at $t=0$ and $m_{z}=0$ $(E=0)$ at $t=\tau$. It is given by

$$
\begin{aligned}
P_{\tau}= & \int \mathcal{D} \xi \int_{E(0)=-M H_{K} \mathcal{V} / 2}^{E(\tau)=0} \mathcal{D} E e^{i \mathcal{S}}, \\
i \mathcal{S}= & -\int_{0}^{\tau} d t\left[i \xi(t) \dot{E}(t)-\mathcal{F}_{G}(0, \xi(t) \hbar \Omega(t) ; \Omega(t))\right. \\
& \left.-\mathcal{F}_{\alpha}(-\xi(t))\right],
\end{aligned}
$$

where we added the SCGF of Gaussian thermal noise,

$$
\mathcal{F}_{\alpha}(\xi)=G_{\alpha} \sin ^{2} \theta(\hbar \Omega)^{2} i \xi(1+i \xi / \beta) .
$$

Since the number of magnetic moments in the nanomagnet $M \mathcal{V} / \mu_{\mathrm{B}}$ is typically large, we utilize the optimalpath approximation. The resulting switching probability acquires the form of Eq. (3) with the switching exponent:

$$
\Delta=-i \mathcal{S}^{*}=-\frac{M \mathcal{V}}{2 \mu_{\mathrm{B}}} \int_{-\gamma H_{K}}^{\Omega^{*}} d \Omega \frac{i \chi^{*}}{\gamma H_{K}} .
$$

When the Gilbert damping is absent $\alpha=0$, $i \chi^{*}=$ $\ln \left[\left(\Gamma_{+-}+\Gamma_{--}\right) /\left(\Gamma_{++}+\Gamma_{-+}\right)\right]$. The solid lines in Fig. 2 are the switching exponents as a function of the bias voltage at a finite temperature and at zero temperature. We find that, at zero temperature below $e V_{\mathrm{th}}=2 \mu_{\mathrm{B}} H_{K}$, the exponent diverges, which means that the switching is completely blocked. This is because the spin flip process $\downarrow \rightarrow \uparrow$ is blocked: $\Gamma_{-+}+\Gamma_{--}=0$. At finite temperature, this divergence disappears and at $\mathrm{eV}=0$, we obtain the Arrhenius law: $\Delta=M H_{K} \mathcal{V} /\left(2 k_{\mathrm{B}} T\right)$. The inset shows results at a finite $\alpha$. We see that the divergence remains. 
Close to the critical voltage, we approximate $i \chi^{*} \approx$ $\left(\hbar \Omega-\hbar \Omega^{*}\right) /\left(k_{\mathrm{B}} T_{\text {eff }}\right)$ and obtain the Arrhenius-like form

$$
\Delta=\frac{M H_{K} \mathcal{V}}{2 k_{\mathrm{B}} T_{\mathrm{eff}}}\left(\frac{V^{*}-V}{V^{*}}\right)^{2},
$$

which quadratically depends on the distance from the critical voltage. The effective temperature,

$$
T_{\text {eff }}=\frac{\sum_{ \pm} G_{\mp}\left(e V \pm \hbar \Omega^{*}\right) \operatorname{coth} \frac{e V \pm \hbar \Omega^{*}}{2 k_{\mathrm{B}} T}+2 k_{\mathrm{B}} T G_{\alpha}}{2 k_{\mathrm{B}}\left(G_{+}+G_{-}+G_{\alpha}\right)},
$$

is reduced to the real temperature $T_{\text {eff }} \approx T$ for high temperatures, $e V, e V_{\mathrm{th}} \ll k_{\mathrm{B}} T$. Then Eq. (17) reproduces the previous result [16, 18]. At zero temperature and $G_{\alpha}=0, T_{\text {eff }} \approx 2 G_{+} G_{-} e V /\left(G_{+}+G_{-}\right)^{2}$, which is proportional to the bias voltage [22], indicating that the spintorque shot noise is the dominant source of fluctuations around the critical voltage. The dashed lines in Fig. 2 show Eq. (17). They fit well for finite temperature or around the critical voltage.

When the volume becomes very small, i.e., $\mathcal{V} \sim \mu_{B} / M$, we have to go beyond the optimal path approximation [34, 35]. In such cases, the time scales of the source of Gaussian noise and that of Poisson noise should be treated carefully [35].

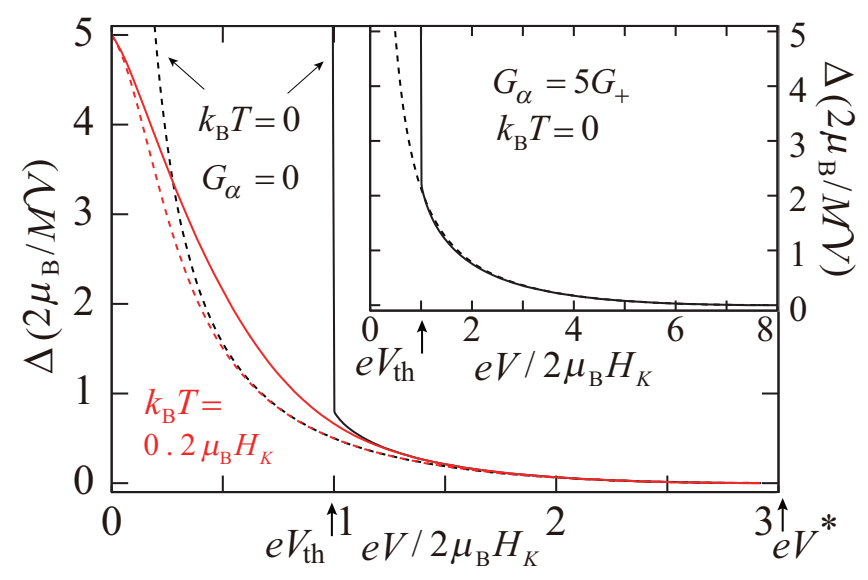

FIG. 2: The bias-voltage dependence of the switching exponent $\Delta$ for $\alpha=0$ and $G_{-}=2 G_{+}$. The two solid lines are for different temperatures $k_{\mathrm{B}} T /\left(2 \mu_{\mathrm{B}} H_{K}\right)=0$ and 0.1 . The inset shows a plot for finite $G_{\alpha}=5 G_{+}$. The dashed lines indicate an Arrhenius-like law, Eq. (17). The critical voltages are $e V^{*} /\left(2 \mu_{\mathrm{B}} H_{K}\right)=3$ and 8 for $G_{\alpha}=0$ and $5 G_{+}$.

Full-counting statistics under the adiabatic pumpingAn electron transferred through the nano-magnet is affected by its precession motion. This scattering process is described by a time-dependent $S$-matrix:

$$
\begin{aligned}
\mathbf{S}(\theta, \phi(t)) & =e^{-i \phi(t) \sigma_{z} / 2} \mathbf{S}(\theta) e^{i \phi(t) \sigma_{z} / 2} \\
\mathbf{S}(\theta) & =\left(\begin{array}{cc}
\mathbf{r}(\theta) & \mathbf{t}^{\prime}(\theta) \\
\mathbf{t}(\theta) & \mathbf{r}^{\prime}(\theta)
\end{array}\right)
\end{aligned}
$$

where the Pauli matrix $\sigma_{z}$ acts in the spin space and $\phi(t) \approx \Omega t+\phi(0) . \mathbf{r}$ and $\mathbf{t}\left(\mathbf{r}^{\prime}\right.$ and $\left.\mathbf{t}^{\prime}\right)$ are $2 \times 2$ matrix of the spin-dependent reflection amplitudes and that of the spin-dependent transmission amplitudes for an incoming wave from the left (right) lead. For example, the element $t_{\sigma^{\prime} \sigma}$ describes an electron transmission from the spin $\sigma$ state in the left lead to the spin $\sigma^{\prime}$ state in the right lead.

The SCGF (8) is expressed by using the $S$-matrix as [25, 26],

$$
\begin{aligned}
& \mathcal{F}_{G}(\lambda, \chi ; \Omega)=\sum_{\ell} \int \frac{d E}{h} \ln \operatorname{det}[\mathbf{1}-\mathbf{f}(E)(\mathbf{1} \\
& \left.\left.-e^{i \boldsymbol{\lambda}+i \chi \sigma_{z} / 2} \mathbf{S}^{\ell}(\theta ; E)^{\dagger} e^{-i \boldsymbol{\lambda}-i \chi \sigma_{z} / 2} \mathbf{S}^{\ell}(\theta ; E)\right)\right],
\end{aligned}
$$

where $\mathbf{S}^{\ell}(E)$ is the $S$-matrix for the $\ell$-th transverse channel. The counting field matrix $\boldsymbol{\lambda}=\operatorname{diag}(\lambda, \lambda, 0,0)$ counts the number of electrons flowing out of the left lead. The precession motion effectively splits the $\uparrow$-spin and $\downarrow$-spin chemical potentials of the 2 leads after the gauge transform, $\mathbf{f}(E)=\operatorname{diag}\left(f_{L}(E+\hbar \Omega / 2), f_{L}(E-\hbar \Omega / 2), f_{R}(E+\right.$ $\left.\hbar \Omega / 2), f_{R}(E-\hbar \Omega / 2)\right)$. The spin-splitting of the chemical potentials is a result of the backaction, which is crucial to be consistent with the FT [33]. It also blocks the spin-flip tunneling process $\downarrow \rightarrow \uparrow$ under the threshold voltage.

Although we considered a simple model, it is also possible to calculate the $S$-matrix using a realistic model. Then, from Eq. (20), we obtain Eq. (13) expressed with general $I_{S z}^{\Omega=0}$ and $p_{\text {pump }}$,

$$
\begin{aligned}
I_{S z}^{\Omega=0}= & \frac{e V}{4 \pi} \sum_{\ell, \sigma=\uparrow, \downarrow}\left(\left|r_{\downarrow \sigma}^{\ell}\left(\theta, \phi ; E_{F}\right)\right|^{2}+\left|t_{\downarrow \sigma}^{\ell}\left(\theta, \phi ; E_{F}\right)\right|^{2}\right. \\
& \left.-\left|r_{\uparrow \sigma}^{\ell}\left(\theta, \phi ; E_{F}\right)\right|^{2}-\left|t_{\uparrow \sigma}^{\ell}\left(\theta, \phi ; E_{F}\right)\right|^{2}\right), \\
p_{\text {pump }}= & \frac{\hbar \Omega^{2}}{4 \pi} \sum_{\ell} \operatorname{tr}\left(\partial_{\phi} \mathbf{S}^{\ell}\left(\theta, \phi ; E_{F}\right) \partial_{\phi} \mathbf{S}^{\ell}\left(\theta, \phi ; E_{F}\right)^{\dagger}\right),
\end{aligned}
$$

in the leading order of $e V$ and $\Omega$. It is straightforward to take the channel mixing scattering into account. Our $p_{\text {pump }}$ reproduces Ref. 27 .

Summary - We demonstrate the switching probability driven by fluctuating non-conservative spin-torque. The theory of the full-counting statistics under the adiabatic pumping enables us to account for the backaction effect and to obtain a distribution of the fluctuating spintorque consistent with the fluctuation theorem for a small engine. We find the threshold voltage $e V_{\mathrm{th}}=2 \mu_{\mathrm{B}} H_{K}$, above which the spin-torque shot noise causes the probabilistic switching. Under the threshold the spin-flip tunneling process is blocked because of the backaction and thus the probabilistic switching is suppressed.

This work was supported by KAKENHI (grant numbers 26400390 and 26220711).

[1] U. Seifert, Rep. Prog. Phys. 75 (2012) 126001. 
[2] M. Esposito, U. Harbola, and S. Mukamel, Rev. Mod. Phys. 81, 1665 (2009).

[3] M. Campisi, P. Hänggi, and M. Talkner, Rev. Mod. Phys. 83, 771 (2011).

[4] J. Tobiska and Yu. V. Nazarov, Phys. Rev. B 72, 235328 (2005).

[5] H. Förster and M Büttiker, Phys. Rev. Lett. 101, 136805 (2008).

[6] K. Saito and Y. Utsumi, Phys. Rev. B 78, 115429 (2008).

[7] D. Andrieux, P. Gaspard, T. Monnai, and S. Tasaki, New J. Phys. 11, 043014 (2009).

[8] Y. Utsumi, H. Imamura, J. Phys.: Conf. Ser. 200, 052030 (2010).

[9] R. López, J.-S. Lim, D. Sánchez, Phys. Rev. Lett. 108, 246603 (2012); J.-S. Lim, D. Sánchez, R. López, arXiv:1208.4746.

[10] N. A. Sinitsyn, J. Phys. A: Math. Theor. 44, 405001 (2011).

[11] M. Campisi, arXiv:1403.8040.

[12] G. Verley, T. Willaert, C. Van den Broeck, and M. Esposito, arXiv:1404.3095

[13] P. Hänggi, P. Talkner, and M. Borkovec, Rev. Mod. Phys. 62, 251 (1990).

[14] J. C. Slonczewski, J. Magn. Magn. Mat. 159, L1-L7 (1996).

[15] D. M. Apalkov, P. B. Visscher, Phys. Rev. B 72, 180405 (2005).

[16] T. Taniguchi, H. Imamura, Phys. Rev. B 83, 054432 (2011).

[17] D. Pinna, A. D. Kent, D. L. Stein, Phys. Rev. B 88, 104405 (2013).

[18] T. Taniguchi, Y. Utsumi, M. Marthaler, D. S. Golubev, H. Imamura, Phys. Rev. B 87, 054406 (2013); T.
Taniguchi, M. Shibata, M. Marthaler, Y. Utsumi, H. Imamura, Appl. Phys. Express 5063009 (2012).

[19] Y. Suzuki, A. A. Tulapurkar, and C. Chappert, in Nanomagnetism and Spintronics, edited by T. Shinjo (Elsevier, Amsterdam, 2009).

[20] Ya. M. Blanter, M. Büttiker, Phys. Rep. 336, 1 (2000).

[21] J. Foros, A. Brataas, Y. Tserkovnyak, G. E. W. Bauer, Phys. Rev. Lett. 95, 016601 (2005).

[22] A. S. Núñez, R. A. Duine, Phys. Rev. B 77, 054401 (2008).

[23] A. L. Chudnovskiy, J. Swiebodzinski, and A. Kamenev, Phys. Rev. Lett 101, 066601 (2008).

[24] L. S. Levitov, H.-W. Lee, and G. B. Lesovik, Journal of Mathematical Physics, 37, 4845 (1996).

[25] A. Andreev, A. Kamenev, Phys. Rev. Lett. 85, 1294 (2000).

[26] A. Kamenev, Field Theory of Nonequilibrium Systems (Cambridge University Press, Cambridge, 2011).

[27] A. Brataas, Y. Tserkovnyak, and G. E. W. Bauer, Phys. Rev. Lett. 101, 037207 (2008); Phys. Rev. B 84, 054416 (2011).

[28] W. F. Brown, Phys. Rev. 130, 1677 (1963).

[29] See Supplemental Material for technical details.

[30] P. Bruno, Phys. Rev. B 52, 411 (1995).

[31] J. C. Slonczewski, Phys. Rev. B 396995 (1989).

[32] See Supplemental Material for technical details.

[33] Y. Utsumi, D. S. Golubev, M. Marthaler, Gerd Schön, and K. Kobayashi, Phys. Rev. B 86, 075420 (2012).

[34] K. Kanazawa, T. Sagawa, H. Hayakawa, Phys. Rev. Lett. 108, 210601 (2012).

[35] K. Kanazawa, T. G. Sano, T. Sagawa, H. Hayakawa, arXiv:1407.5267.

\section{SUPPLEMENTAL MATERIAL}

Technical details of derivations of a scattering matrix, a scaled cumulant generating function and a switching exponent.

\section{Scattering matrix and the scaled cumulant generating function}

We derive the $S$-matrix of the ferromagnet/ferromagnetic insulator/normal metal structure. We take the $z$-axis perpendicular to the interface and assume translational invariance in the $x$ and $y$ directions. The Schrödinger equation is

$$
\left(-\frac{\hbar^{2}}{2 m} \nabla^{2}+U(z)\right) \psi(x, y, z)=E \psi(x, y, z), \quad U(z)=\left\{\begin{array}{cc}
\mu_{\mathrm{B}} H_{\mathrm{m} L} \sigma_{z} / 2 & (z<0) \\
U_{0}+\mu_{\mathrm{B}} H_{\mathrm{m}} \mathbf{m} \cdot \vec{\sigma} & (0 \leq z<d) \\
0 & (d \leq z)
\end{array}\right.
$$

where $\vec{\sigma}=\left(\sigma_{x}, \sigma_{y}, \sigma_{z}\right)$ is the Pauli matrix vector. The thickness of the ferromagnetic insulator is $d$ and $U_{0}>0$ is the potential barrier height. The molecular (exchange) fields in the ferromagnetic lead and in the ferromagnetic insulator are $H_{\mathrm{m} L}$ and $H_{\mathrm{m}}$, respectively. The wave function is written as $\psi(x, y, z)=2 \sin \left(\pi \ell_{x} x / L\right) \sin \left(\pi \ell_{y} y / L\right) \psi(z) / L$ where the contact area is $0 \leq x, y \leq L$ ( $\ell_{y}$ and $\ell_{z}$ are non-negative integers). In the $z$ direction, the Schrödinger equation reads

$$
\left(-\frac{\hbar^{2}}{2 m} \frac{\partial^{2}}{\partial z^{2}}+U(z)\right) \psi_{\ell}(z)=E_{\ell} \psi_{\ell}(z), \quad E_{\ell}=E-\frac{\hbar^{2} \pi^{2}}{2 m L^{2}}\left(\ell_{y}^{2}+\ell_{z}^{2}\right),
$$

where we introduced the channel index $\ell=\left(\ell_{x}, \ell_{y}\right)$. The wave number of an electron with the energy $E$ is $k_{\sigma}=$ $\sqrt{2 m\left(E_{\ell}-\sigma \mu_{\mathrm{B}} H_{\mathrm{m} L}\right)} / \hbar$ in the ferromagnetic lead $(z<0), i \kappa_{\sigma}=\sqrt{2 m\left(E_{\ell}-U_{0}-\sigma \mu_{\mathrm{B}} H_{\mathrm{m}} / 2\right)} / \hbar$ in the ferromagnetic 
insulator $(0<z<d)$ and $k=\sqrt{2 m E_{\ell}} / \hbar$ in the normal metal lead $(d<z)$. The $S$-matrix in the leading order of $e^{-\kappa_{\sigma} d}$ is calculated as

$$
\mathbf{S}(\theta)=\left(\begin{array}{cc}
\mathbf{P} & \mathbf{0} \\
\mathbf{0} & \mathbf{P}^{\prime}
\end{array}\right)\left(\begin{array}{cc}
-\mathbf{1}-\left(i \mathbf{A}+\boldsymbol{\tau}^{\dagger} \boldsymbol{\tau} / 2\right) & \boldsymbol{\tau}^{\dagger} \\
\boldsymbol{\tau} & \mathbf{1}+\left(i \mathbf{A}+\boldsymbol{\tau} \boldsymbol{\tau}^{\dagger} / 2\right)
\end{array}\right)\left(\begin{array}{cc}
\mathbf{P} & \mathbf{0} \\
\mathbf{0} & \mathbf{P}^{\prime}
\end{array}\right) .
$$

where an Hermite matrix $\mathbf{A}^{\dagger}=\mathbf{A}$ is not relevant for our model. Further, we neglect $H_{\mathrm{m}}$ except when it appears in the exponent of $e^{-\kappa_{\sigma} d}$. Then we obtain the following $2 \times 2$ matrix of the spin-dependent transmission amplitude:

$$
\boldsymbol{\tau}=\frac{1}{2}\left(\begin{array}{cc}
\tau_{\uparrow \uparrow}+\tau_{\downarrow \uparrow}+\left(\tau_{\uparrow \uparrow}-\tau_{\downarrow \uparrow}\right) \cos \theta & \left(\tau_{\uparrow \downarrow}-\tau_{\downarrow \downarrow}\right) \sin \theta \\
\left(\tau_{\uparrow \uparrow}-\tau_{\downarrow \uparrow}\right) \sin \theta & \tau_{\uparrow \downarrow}+\tau_{\downarrow \downarrow}+\left(\tau_{\uparrow \downarrow}-\tau_{\downarrow \downarrow}\right) \cos \theta
\end{array}\right), \quad \tau_{\sigma \sigma^{\prime}}=4 e^{-\kappa_{\sigma} d} \sqrt{\frac{\kappa_{0} k}{\kappa_{0}^{2}+k^{2}} \frac{\kappa_{0} k_{\sigma^{\prime}}}{\kappa_{0}^{2}+k_{\sigma^{\prime}}^{2}} .}
$$

$2 \times 2$ sub-matrices $\mathbf{P}, \mathbf{P}^{\prime}$ become diagonal and $(\sigma, \sigma)$ component of $\mathbf{P}^{2}$ and $\mathbf{P}^{\prime 2}$ are $\left(\kappa_{0}+i k_{\sigma}\right) /\left(\kappa_{0}-i k_{\sigma}\right)$ and $-i\left(\kappa_{0}+i k\right) /\left(\kappa_{0}-i k\right)$, where $\kappa_{0}=\sqrt{2 m\left(U_{0}-E_{\ell}\right)} / \hbar$.

We insert the $S$-matrix (23) into Eq. (20) in the main text:

$$
\mathcal{F}_{G}(\lambda, \chi ; \hbar \Omega)=\rho_{\|} \int d E_{\|} \int \frac{d E}{h} \ln \operatorname{det}\left[\mathbf{1}+\mathbf{f}(E)\left(e^{i \boldsymbol{\lambda}+i \chi \sigma_{z} / 2} \mathbf{S}\left(E-E_{\|}, \theta\right)^{\dagger} e^{-i \boldsymbol{\lambda}-i \chi \sigma_{z} / 2} \mathbf{S}\left(E-E_{\|}, \theta\right)-\mathbf{1}\right)\right],
$$

where $\rho_{\|}=2 \pi m L^{2} / h^{2}$ is the DOS of the transverse channel. Since the energy dependence of $\tau_{\sigma \sigma^{\prime}}$ is small around the Fermi energy $E_{\mathrm{F}}$, it is possible to approximate $\tau_{\sigma \sigma^{\prime}}\left(E-E_{\|}\right) \approx \tau_{\sigma \sigma^{\prime}}\left(E_{F}\right) \exp \left(-E_{\|} /(2 \delta)\right)$, where $\delta^{-1}=2 d \partial \kappa_{0}\left(E_{\ell}=\right.$ $\left.E_{F}\right) / \partial E_{\ell}$. After performing the integral and expanding up to the leading order in $e^{-\kappa_{\sigma} d}$, we obtain Eq. (9) in the main text. The conductances are

$$
\begin{aligned}
G_{+} & =\frac{1}{h} \rho_{\|} \delta\left|\tau_{\downarrow \downarrow}\left(E_{F}\right)-\tau_{\uparrow \downarrow}\left(E_{F}\right)\right|^{2}, \\
G_{-} & =\frac{1}{h} \rho_{\|} \delta\left|\tau_{\uparrow \uparrow}\left(E_{F}\right)-\tau_{\downarrow \uparrow}\left(E_{F}\right)\right|^{2}, \\
G_{\mathrm{P}} & =\frac{1}{h} \rho_{\|} \delta\left(\left|\tau_{\uparrow \uparrow}\left(E_{F}\right)\right|^{2}+\left|\tau_{\downarrow \downarrow}\left(E_{F}\right)\right|^{2}\right), \\
G_{\mathrm{AP}} & =\frac{1}{h} \rho_{\|} \delta\left(\left|\tau_{\uparrow \downarrow}\left(E_{F}\right)\right|^{2}+\left|\tau_{\downarrow \uparrow}\left(E_{F}\right)\right|^{2}\right) .
\end{aligned}
$$

The reversal of the magnetic moments $\mathbf{M} \rightarrow-\mathbf{M}$ and $\mathbf{M}_{L} \rightarrow-\mathbf{M}_{L}$, which corresponds to $H_{\mathrm{m}} \rightarrow-H_{\mathrm{m}}$ and $H_{\mathrm{m} L} \rightarrow-H_{\mathrm{m} L}$, changes the tunneling amplitude to $\tau_{\sigma \sigma^{\prime}} \rightarrow \tau_{\sigma^{\prime} \sigma}$ and thus the conductances to $G_{+} \leftrightarrow G_{-}$.

\section{Switching exponent}

We analyze the Langevin equation (6) in the main text by exploiting the Martin-Siggia-Rose approach (see Section 4 in Ref. 1). We first discretize time $\tau$ into $N=\tau / \Delta t$ steps. For now, we neglect the equilibrium power dissipation $p_{\alpha}$. The variation of the energy during a short time step from $t_{j}=\Delta t j$ to $t_{j+1}$ is

$$
E_{j+1}-E_{j} \approx \hbar \Omega_{j} s_{j}, \quad s_{j}=\int_{t_{j}}^{t_{j+1}} d t \overline{I_{S z}(t)},
$$

where $E_{j}=E\left(t_{j}\right)$ and $\Omega_{j}=\Omega\left(\left(E_{j+1}+E_{j}\right) / 2\right)$. The stochastic variable $s_{j}$ is distributed according to the joint probability distribution Eq. (8) described in the main text. The conditional joint probability to find $E_{j}$ at time $t_{j}$ and $E_{j+1}$ at $t_{j+1}$ accompanied by $n_{j}$ electron transmission is given by

$$
\begin{aligned}
P_{\Delta t}\left(n_{j}, E_{j+1} \mid E_{j}\right) & =\int d \epsilon_{S j} \delta\left(E_{j+1}-E_{j}-\epsilon_{S j}\right) \sum_{s, n} P_{\Delta t}\left(n, s ; \Omega_{j}\right) \delta\left(\epsilon_{S j}-\hbar \Omega_{j} s\right) \delta_{n_{j}, n} \\
& =\int_{-\pi}^{\pi} \frac{d \lambda_{j}}{2 \pi} \int \frac{d \xi_{j}}{2 \pi} e^{-i \lambda_{j} n_{j}-i \xi_{j}\left(E_{j+1}-E_{j}\right)+\mathcal{F}_{G}\left(\lambda_{j}, \hbar \Omega_{j} \xi_{j} ; \Omega_{j}\right) \Delta t} .
\end{aligned}
$$

Then the conditional joint probability to find $E(0)$ at $t=0$ and $E(\tau)$ at $\tau$ accompanied by $n$ electron transmission is calculated by accumulating joint probabilities for short time steps as follows:

$$
\begin{aligned}
P_{\tau}(n, E(\tau) \mid E(0)) & =\sum_{n_{0}, \cdots, n_{N-1}} \int d E_{1} \cdots d E_{N-1} P_{\Delta t}\left(n_{N-1}, E_{N} \mid E_{N-1}\right) \cdots P_{\Delta t}\left(n_{0}, E_{1} \mid E_{0}\right) \delta_{n, \sum_{j=0}^{N-1} n_{j}} \\
& =\int_{-\pi}^{\pi} \frac{d \lambda}{2 \pi} \int \frac{d \xi_{0}}{2 \pi} \cdots \frac{d \xi_{N-1}}{2 \pi} \int d E_{1} \cdots d E_{N-1} e^{\sum_{j=0}^{N-1}\left[-i \xi_{j}\left(E_{j+1}-E_{j}\right)+\mathcal{F}_{G}\left(\lambda, \xi_{j} \hbar \Omega_{j} ; \Omega_{j}\right) \Delta t\right]-i \lambda n} .
\end{aligned}
$$


We can prove the detailed FT by Jarzynski [2] based on the FT (12) in the main text along the same line of the proof in Ref. 3 ,

$$
P_{\tau}(n, E(\tau) \mid E(0)) / P_{R, \tau}(-n, E(0) \mid E(\tau))=e^{\beta[n e V-E(\tau)+E(0)]},
$$

In order to account for the equilibrium power dissipation, we can just replace $\mathcal{F}_{G}$ with $\mathcal{F}_{G}(\lambda, \xi \hbar \Omega ; \Omega)+\mathcal{F}_{\alpha}(-\xi)$. Further, for calculating the switching rate, we can sum over $n, P_{\tau}(E(\tau) \mid E(0)) \equiv \sum_{n} P_{\tau}(n, E(\tau) \mid E(0))$. Then in the continuous limit, $\Delta t \rightarrow 0$, we obtain the path-integral form Eq. (15) in the main text.

Since $E, G_{\alpha} \propto \mathcal{V}=L^{2} d$ and $\mathcal{F}_{G} \propto L^{2} / d$, for a modestly large nano-magnet, it is possible to perform the optimal path approximation [1, 4, 6]. Namely, from the variational principle, we derive the "canonical equation of motion":

$$
\dot{E}=\frac{\partial \mathcal{F}}{\partial(i \xi)}, \quad i \dot{\xi}=-\frac{\partial \mathcal{F}}{\partial E}
$$

The "momenta" $i \xi$ measures the strength of the fluctuations. $i \xi=0$ corresponds to the noiseless case, which is always an optimal path. The equation of motion possesses the integral of motion, which is the "energy," $\mathcal{F}$. Since the normalization condition ensures $\mathcal{F}(\xi=0 ; \Omega)=0$, the optimal paths always satisfy $\mathcal{F}(\xi ; \Omega)=0$.

We are interested in an optimal path that starts from $(E, i \xi)=\left(-M H_{K} \mathcal{V} / 2,0\right)$ and reaches $(E, i \xi)=(0,0)$. For $\alpha=0$, we find 4 simple solutions satisfying $\mathcal{F}(\xi ; \Omega)=0$ :

$$
\hbar \Omega(E)= \pm 2 \mu_{\mathrm{B}} H_{K}, \quad i \hbar \Omega(E) \xi^{*}=\ln \frac{\Gamma_{+-}+\Gamma_{--}}{\Gamma_{++}+\Gamma_{-+}}, \quad i \xi^{*}=0 .
$$

Figure 3 (a) shows the optimal paths. The horizontal axis is $\Omega=-\sqrt{-2 \gamma^{2} H_{K} E /(M \mathcal{V})}$ and thus $E=-M H_{K} \mathcal{V} / 2$ and $E=0$ correspond to $\hbar \Omega=-2 \mu_{\mathrm{B}} H_{K}$ and $\hbar \Omega=0$, respectively. Arrows indicate the directions of motion determined from Eq. (35). The initial state is at M, i.e., $(\hbar \Omega, i \xi \hbar \Omega)=\left(-2 \mu_{\mathrm{B}} H_{K}, 0\right)$, and the final state is at T, i.e., $(\hbar \Omega, i \xi \hbar \Omega)=(0,0)$. The optimal path is $\mathrm{M} \rightarrow \mathrm{M}^{\prime} \rightarrow \mathrm{U} \rightarrow \mathrm{T}$, where the intermediate state $\mathrm{U}$ is $(\hbar \Omega, i \xi \hbar \Omega)=\left(\hbar \Omega^{*}, 0\right)$. The action along this path is calculated as

$$
i \mathcal{S}^{*}=-\int_{-M H_{K} \mathcal{V} / 2}^{E\left(\Omega^{*}\right)} d E\left(i \xi^{*}\right)=\frac{M \mathcal{V}}{2 \mu_{\mathrm{B}} \gamma H_{K}} \int_{-2 \mu_{\mathrm{B}} H_{K}}^{\Omega^{*}} d \Omega i \xi^{*} \hbar \Omega \equiv-\Delta .
$$

The integral $\int d \Omega i \xi^{*} \hbar \Omega$ gives the area of the shaded region in Fig. 3 (a). This equation leads to Eq. (16) in the main text and the switching probability up to the single instanton contribution, $P_{\tau}\left(E(\tau)=0 \mid E(0)=-M H_{K} \mathcal{V} / 2\right) \approx e^{-\Delta}$.

At zero temperature, the integral (37) can be performed easily. For $e V_{\mathrm{th}}=2 \mu_{\mathrm{B}} H_{K}<e V<e V^{*}$, we obtain

$$
i \mathcal{S}^{*}=\frac{M \mathcal{V}}{2 \mu_{\mathrm{B}}}\left\{\ln \frac{G_{-}\left(e V-2 \mu_{\mathrm{B}} H_{K}\right)}{G_{+}\left(e V+2 \mu_{\mathrm{B}} H_{K}\right)}+\frac{e V}{2 \mu_{\mathrm{B}} H_{K}} \ln \frac{4 G_{+} G_{-}(e V)^{2}}{\left(G_{+}+G_{-}\right)^{2}\left[(e V)^{2}-\left(2 \mu_{\mathrm{B}} H_{K}\right)^{2}\right]}\right\} .
$$

For $e V<e V_{\text {th }}$, it diverges to $i \mathcal{S}^{*}=-\infty$, which means that the switching is completely blocked. Figure 3 (b) shows the optimal path at $e V=e V_{\mathrm{th}}$. M' approaches $(\hbar \Omega, i \xi \hbar \Omega)=(0,-\infty)$ in the limit of zero temperature, and the area of the shaded region diverges. For $G_{\alpha} \neq 0$, the optimal path is modified and we determine it numerically.

With increasing bias voltage, the shaded area decreases and eventually $\mathrm{M}^{\prime}$ and $\mathrm{U}$ meet at M [Fig. 3 (c)]. The exponent and the switching probability become $i \mathcal{S}^{*}=0$ and $P_{\tau} \approx 1$. This critical condition is achieved at $\hbar \Omega^{*}=$ $-2 \mu_{\mathrm{B}} H_{K}$, which is equivalent to the balance condition $\left\langle p_{S}\left(\Omega^{*}\right)\right\rangle=\left\langle p_{\alpha}\left(\Omega^{*}\right)\right\rangle$. Around the critical point (for $\left.G_{\alpha} \neq 0\right)$, we can expand $\xi^{*}$ around $\Omega=\Omega^{*}$ and $\xi=0$, up to the lowest order as

$$
i \xi^{*} \hbar \Omega \approx \frac{\hbar \Omega-\hbar \Omega^{*}}{k_{\mathrm{B}} T_{\mathrm{eff}}} .
$$

By plugging this expression into Eq. (37), we obtain Eq. (17) in the main text.

[1] A. Kamenev, Field Theory of Nonequilibrium Systems (Cambridge University Press, Cambridge, 2011).

[2] C. Jarzynski, J. Stat. Phys. 98, 77 (2000).

[3] Y. Utsumi, O. Entin-Wohlman, A. Aharony, T. Kubo, and Y. Tokura, Phys. Rev. B 89, 205314 (2014).

[4] J. Tobiska and Yu. V. Nazarov, Phys. Rev. Lett. 93, 106801 (2004).

[5] E. V. Sukhorukov and A. N. Jordan, Phys. Rev. Lett. 98, 136803 (2007).

[6] L. Billings, I. B. Schwartz, M. McCrary, A. N. Korotkov, and M. I. Dykman, Phys. Rev. Lett. 104, 140601 (2010). 


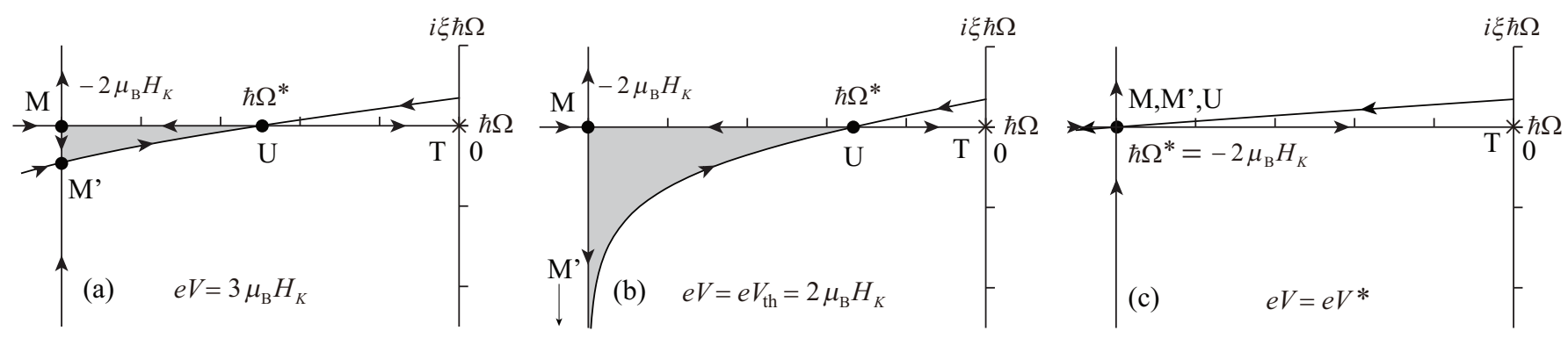

FIG. 3: The optimal paths (a) for $e V=3 \mu_{\mathrm{B}} H_{K}$, (b) for the threshold voltage $e V=e V_{\mathrm{th}}=2 \mu_{\mathrm{B}} H_{K}$ and (c) for the critical voltage $e V=e V^{*}=6 \mu_{\mathrm{B}} H_{K}$. The parameters are as follows: $G_{-}=2 G_{+}, G_{\alpha}=0$ and $k_{\mathrm{B}} T=0$. 